\title{
White matter tract integrity in treatment-resistant gambling disorder
}

Samuel R. Chamberlain, Katherine Derbyshire, Richard E. Daws, Brian L. Odlaug,

Eric W. Leppink and Jon E. Grant

\section{Background}

Gambling disorder is a relatively common psychiatric disorder recently re-classified within the DSM-5 under the category of 'substance-related and addictive disorders'.

\section{Aims \\ To compare white matter integrity in patients with gambling disorder with healthy controls; to explore relationships between white matter integrity and disease severity in gambling disorder \\ Method \\ In total, 16 participants with treatment-resistant gambling disorder and 15 healthy controls underwent magnetic resonance imaging (MRI). White matter integrity was analysed using tract-based spatial statistics.}

\section{Results}

Gambling disorder was associated with reduced fractional anisotropy in the corpus callosum and superior longitudinal fasciculus. Fractional anisotropy in distributed white matter tracts elsewhere correlated positively with disease severity.

\section{Conclusions}

Reduced corpus callosum fractional anisotropy is suggestive of disorganised/damaged tracts in patients with gambling disorder, and this may represent a trait/vulnerability marker for the disorder. Future research should explore these measures in a larger sample, ideally incorporating a range of imaging markers (for example functional MRI) and enrolling unaffected first-degree relatives of patients.

\section{Declaration of interest}

J.E.G. has received research grants from Forest and Roche Pharmaceuticals. S.R.C. consults for Cambridge Cognition. B.L.O. consults for $H$. Lundbeck A/S.

\section{Copyright and usage}

(c) The Royal College of Psychiatrists 2016.
Gambling is a commonplace recreational activity dating back thousands of years, yet for between 1 and $3 \%$ of the population, it develops into a dysfunctional pattern of behaviour. ${ }^{1}$ In those who develop gambling disorder, the behaviour is associated with significant personal, financial and relationship difficulties ${ }^{2,3}$ as well as elevated rates of suicide. ${ }^{4}$ Despite the morbidity and mortality associated with gambling disorder, brain architecture in gambling disorder has received limited research attention. To the knowledge of the authors, only two studies to date have explored white matter tract integrity in gambling disorder. Joutsa and colleagues found reduced white matter integrity (reduced fractional anisotropy) in multiple regions, using tract-based spatial statistics (TBSS), in 12 patients with gambling disorder compared with 12 controls. ${ }^{5}$ Regions of abnormality included the corpus callosum, cingulum, superior longitudinal fascicle, anterior limb of internal capsule, anterior thalamic radiation, inferior longitudinal fascicle and the uncinate/inferior frontooccipital fascicle. The authors found no significant grey matter volume abnormalities in the patients. A separate study focusing on the corpus callosum (region-of-interest rather than wholebrain approach) found reductions of white matter integrity (reduced fractional anisotropy) in sectors of the corpus callosum in 19 participants with gambling disorder $v .19$ controls. $^{6}$

Thus, tentative evidence for white matter abnormalities in gambling disorder has been reported in two previous studies. Neither study, however, explored possible relationships between white matter abnormalities and disease severity, or used a treatment-resistant sample. Although little is known about the clinical differences between patients with non-treatment-resistant and treatment-resistant gambling disorder, research into other psychiatric disorders such as depression indicate that the illness is more severe in those patients whose condition is treatment resistant and they place a larger burden on the healthcare system than those patients with non-treatment-resistant depression. ${ }^{7}$ We therefore explored white matter in people with treatment-resistant gambling disorder $v$. healthy controls, and the relationships between white matter parameters and disease severity in gambling disorder. Based on the previous literature, we hypothesised that those with gambling disorder would show reduced white matter integrity in the corpus callosum.

\section{Method}

\section{Participants}

Participants with gambling disorder (the gambling disorder group) were recruited from an out-patient psychiatry clinic and from media advertisements on the basis of meeting DSM-5 criteria (i.e. minimum of four of nine DSM-5 criteria). ${ }^{8}$ Inclusion criteria across all participants were: 18-65 years old, right-handedness, no current or past month use of psychotropic medications, not pregnant or breastfeeding, a negative urine drug screen and no contraindication to magnetic resonance imaging (MRI). Participants with gambling disorder were required to be treatment resistant, which we defined as having completed at least six sessions of cognitive-behavioural therapy (CBT) and a trial of naltrexone for their gambling disorder with limited or no success. CBT was delivered by trained psychologists using a manualised treatment. Treatment consisted of six sessions (psychoeducation, motivational enhancement, functional analysis and behavioural strategies, coping with gambling urges and changing irrational thinking, imaginal desensitisation and relapse prevention). ${ }^{9}$ Naltrexone treatment comprised at least 12 weeks at an adequate treatment dose (defined as $50 \mathrm{mg}$ /day or higher). ${ }^{10}$ Limited or no success was operationalised as a less than $35 \%$ reduction from baseline in total disease severity scores, measured using the 
Yale-Brown Obsessive Compulsive Scale modified for Pathological Gambling (PG-YBOCS $\left.{ }^{11}\right) .{ }^{12}$ Previous literature indicates that naltrexone treatment is unsuccessful in approximately $25 \%$ of cases $^{13}$ and that CBT is ineffective in approximately $7-32 \%$ of cases. ${ }^{14}$ Exclusion criteria for patients included any current psychotic, bipolar or substance use disorder, or any lifetime substance use disorder.

Healthy controls (the control group) were recruited via word of mouth or media advertisements. They were required to have no current or lifetime psychiatric disorders and no family history of either substance or behavioural addiction. Family members were not interviewed directly and report of family history was based on self-report by the controls.

\section{Procedures}

All participants underwent a semi-structured psychiatric evaluation by a board-certified psychiatrist. Gambling symptomatology was assessed using the Structured Clinical Interview for Pathological Gambling (SCI-PG), ${ }^{12}$ which was adapted for DSM-5, the PG-YBOCS (clinician-administered scale of gambling severity for the past week with two subscales: gambling behaviour and gambling urges), ${ }^{11}$ and the Gambling Symptom Assessment Scale (G-SAS, self-report scale of gambling severity for the past week). ${ }^{15}$ Psychiatric comorbidity was assessed using the Mini International Neuropsychiatric Interview (MINI). ${ }^{16}$ Other assessments included the Hamilton Rating Scale for Depression (HRSD),${ }^{17}$ the Sheehan Disability Scale ${ }^{18}$ and the Quality of Life Inventory (QoLI). ${ }^{19}$

All participants underwent a comprehensive safety assessment for magnetic resonance imaging (MRI). On completion of these assessments, structural imaging was conducted on the same day using a 3-Tesla (3T) Siemen's system. All study procedures were conducted in accordance with the ethical standards established by the latest version of the Declaration of Helsinki. A full institutional review board approved the study and all consent procedures. After complete description of the study to the participants, they provided written informed consent. No secondary consent (i.e. parent or guardian) was allowed for this study.

\section{Data analysis}

Demographic characteristics

Between-group differences in salient demographic characteristics were explored using independent sample $t$-tests, with statistical significance defined as $P<0.05$ uncorrected.

\section{Diffusion MRI scans and preprocessing}

Imaging data were acquired using a 3T system. Diffusion-weighted imaging data were obtained ( 25 directions) with slice thickness of $4 \mathrm{~mm}$, temporal resolution of $12 \mathrm{~s}$, echo time $(\mathrm{TE})=93 \mathrm{~ms}$, matrix size of $128 \times 128$, field of view $(\mathrm{FoV})=30 \times 24 \mathrm{~cm}^{2}$, and a B-value of $1000 \mathrm{~s} / \mathrm{mm}^{2}$. One volume without diffusion weighting $(b=0)$ was also acquired. To provide a reference, axial three-dimensional $T_{1}$-weighted images were obtained using a spoiled-gradient recall sequence with slice thickness of $2 \mathrm{~mm}$, temporal resolution of $33 \mathrm{~ms}, \mathrm{TE}=3 \mathrm{~ms}, \mathrm{FoV}=24 \mathrm{~cm}$, flip angle of $40^{\circ}$ and matrix size of $256 \times 256$

Individuals' raw diffusion and $b=0$ volumes were concatenated into a single four-dimensional file prior to being corrected for geometric and eddy current distortions, and subject motion. ${ }^{20,21}$ Following this, affine transformations were used to warp each diffusion volume within each participant's time series to the $b=0$ reference image. Participant-specific brain masks were created to demarcate voxels to be modelled with the diffusion tensor. Tensors were fit to each voxel within each participant's brain mask using a least squares weighted approach. From this, whole brain maps of fractional anisotropy, mean diffusivity and Eigenvalue 1, 2 and 3 were generated. Fractional anisotropy is a measure of directionality of water diffusion, whereas mean diffusivity is the mean of the three Eigenvalues and provides a measure of average water mobility.

\section{Tract-based spatial statistics (TBSS)}

Analyses were conducted using previously validated procedures as implemented in TBSS software. ${ }^{20,21}$ Voxel outliers from the fourdimensional files were removed and each participant's fractional anisotropy images were then aligned. This process selected the most representative target image of the group, which was then affine aligned into Montreal Neurological Institute (MNI) space. Subsequently, all of the other images were registered into MNI space based on the non-linear transformations that were carried out on the representative image. From this a mean fractional anisotropy image was created and thinned to form a white matter skeleton that represents the centres of all tracts common to the group.

\section{Group-level and correlational analyses}

Voxel-wise statistics were used to test for group differences in measures of diffusivity using a significance threshold of $P<0.05$. Each participant's aligned fractional anisotropy data were projected onto this skeleton and the resulting data were fed into voxel-wise cross-participant statistics. Group differences in each measure of diffusion were tested for using a permutation-based analysis with 10000 permutations. The resulting statistical maps were corrected for using threshold-free cluster enhancement (TFCE) and family-wise error (FEW, $P<0.05$ ). Unless stated, age was mean-centred and was included as a covariate of no interest. This process was repeated within the gambling disorder group to investigate whether gambling measures correlated with voxel-wise measurement of diffusion. Variables were meancentred and each analysis included age as a covariate of no interest. For visualisation, voxels showing significant differences were filled. All maps were rendered using the lowest probability values possible.

\section{Results}

Demographic and clinical characteristics of the sample are shown in Table 1. A total of 16 patients with DSM-5 gambling disorder (mean age 47.0 years, s.d. $=13.9$ ) and 15 healthy controls (mean age 32.9 years, s.d. $=14.7$ ) were enrolled and underwent brain imaging. The gambling disorder group were significantly older than the controls $(P=0.007)$. There were no other significant demographic differences between groups. The gambling disorder group had a mean PG-YBOCS score of 24.2 (s.d.=4.7) and a mean G-SAS score of 33.8 (s.d. = 7.1) both of which corresponded to a severe gambling disorder. The gambling disorder group had a low quality of life (mean total score of 29.8, s.d. =12.6) according to the QoLI but relatively low depression scores on the HRSD (mean total score 8.3, s.d. $=4.5$ ).

\section{Between-group differences in measures of diffusion}

TBSS analysis demonstrated significantly reduced fractional anisotropy in the gambling disorder group $v$. the control group (Fig. 1, shown in red). This cluster of reduced fractional anisotropy in the gambling disorder group is interpreted as corpus callosal fibres including the middle and middle posterior body of the 


\begin{tabular}{|c|c|c|c|c|c|}
\hline & $\begin{array}{c}\text { Gambling } \\
\text { disorder group } \\
(n=16)\end{array}$ & $\begin{array}{l}\text { Control } \\
\text { group } \\
(n=15)\end{array}$ & $t$-test & $\chi^{2}$ & $P$ \\
\hline Age, years: mean (s.d.) & $47.4(13.7)$ & $32.5(14.9)$ & 2.889 & & 0.007 \\
\hline Female, $n(\%)$ & $10(62.5)$ & $12(80)$ & & 1.151 & 0.283 \\
\hline Ethnicity, White: $n(\%)$ & $13(81.3)$ & $14(93.3)$ & & 1.006 & 0.316 \\
\hline Education, $\geqslant$ college: $n(\%)$ & $8(50)$ & $11(73.3)$ & & 1.777 & 1.823 \\
\hline Hamilton Rating Scale for Depression, mean (s.d.) & $8.3(4.5)$ & & & & \\
\hline \multicolumn{6}{|c|}{ Yale-Brown Obsessive Compulsive Scale for Pathological Gambling, mean (s.d.) } \\
\hline Urges & $12.2(2.8)$ & & & & \\
\hline Behaviours & $12(3.6)$ & & & & \\
\hline Total score & $24.2(4.7)$ & & & & \\
\hline Gambling Symptom Assessment Scale, mean (s.d.) & $33.8(7.1)$ & & & & \\
\hline Sheehan Disability Scale, mean (s.d.) & $17.8(8.2)$ & & & & \\
\hline Quality of life score, mean (s.d.) & $29.8(12.6)$ & & & & \\
\hline
\end{tabular}

corpus callosum, which either extend from the corpus callosal proper or is also present in an independent aspect of the superior longitudinal fasciculus/cortico spinal tract. When age was not included as a covariate in the analysis, this difference in these regions remained significant, but also extended across a broader range of areas (Fig. 1, shown in blue). No significant group differences were observed based on mean diffusivity.

\section{Relationships with gambling measures in the gambling disorder group}

In the gambling disorder group there was a significant positive correlation $(P<0.03)$ between the urge subscale of the PG-YBOCS and fractional anisotropy in distributed white matter tracts (Fig. 2); and a significant positive correlation $(P<0.01)$ between G-SAS and fractional anisotropy within large right hemisphere white matter tracts (Fig. 3). Representative scatter plots are provided in online Figs DS1 and DS2 respectively. No significant correlations were found between fractional anisotropy and the gambling behaviour subscale of the PG-YBOCS nor were significant correlations found between mean diffusivity and any of these clinical measures.

\section{Discussion}

\section{Main findings}

To our knowledge, this is the first study to examine white matter tract integrity in people with treatment-resistant gambling disorder v. controls. Gambling disorder was associated with significant reductions of fractional anisotropy in the corpus callosum, suggestive of damaged and/or disorganised white matter. Additionally, fractional anisotropy was positively correlated with symptom severity in other white matter regions in the gambling disorder group. No group differences were identified with respect to mean diffusivity, suggesting that the white matter abnormalities were more related to microarchitecture of tracts (i.e. tract integrity) than local lesions.

\section{Comparison with findings from other studies}

Our finding of reduced fractional anisotropy in the corpus callosum (Fig. 1) is in broad agreement with reductions in fractional anisotropy seen in this region in two previous gambling disorder studies. Yip and colleagues used a region-of-interest approach, exploring white matter in distinct sectors of the corpus callosum. They found reduced fractional anisotropy values in the left body and left and right genu in particular, $v$. controls. ${ }^{6}$ By contrast, Joutsa et al used a whole-brain approach (TBSS) as we did here; the authors reported reduced fractional anisotropy not just in the corpus callosum but also elsewhere, in patients with gambling disorder compared with controls. ${ }^{5}$ It is unclear why our group-level differences are more restricted in anatomical scope as compared with this second study. There were several differences between the current study and Joutsa et al: ${ }^{5}$ we included men and women, whereas their study was men only; our study had a larger sample size; and finally, age differed between groups in our study but not in their study.

\section{Interpretation of our findings}

Our data add to a growing body of evidence implicating aberrant white matter tracts of the corpus callosum across impulsive and compulsive disorders. Similar abnormalities of fractional anisotropy in this region have been found in obsessive-compulsive disorder, ${ }^{22}$ kleptomania, ${ }^{23}$ trichotillomania ${ }^{24}$ and in various substance use disorders. ${ }^{25}$ The white matter of the corpus callosum enables communication and integration of information across distributed grey matter nodes, and is heavily implicated in cognitive functioning, including in top-down inhibitory control. ${ }^{26}$ Thus it is conceivable, but has yet to be demonstrated, that diminished integrity of the corpus callosum in gambling disorder may underpin some of the cognitive deficits observed in gambling disorder across studies. ${ }^{27}$

Whereas reduced fractional anisotropy was found in the corpus callosum, fractional anisotropy was positively (rather than negatively) associated with worse symptom severity in the gambling disordered group, across a broad swathe of other white matter regions (Figs 2 and 3). One interpretation of these findings is disorganised white matter tracts in the corpus callosum may represent a vulnerability marker (or trait marker) in gambling disorder, which does not relate directly to symptom severity, whereas more organised white matter tracts elsewhere are associated with worse symptom severity and so correspond to the state nature of the illness. Given that the positive correlation between symptom severity and fractional anisotropy was contrary to our expectations, replication will be important in future work to confirm or refute this relationship. It is not possible, on the basis of the current study, to establish whether this positive relationship between symptom severity and higher fractional anisotropy in certain regions is intrinsic to the illness, or rather represents some form of adaptive brain changes/compensatory mechanisms. These issues should be further addressed in larger 
(a)
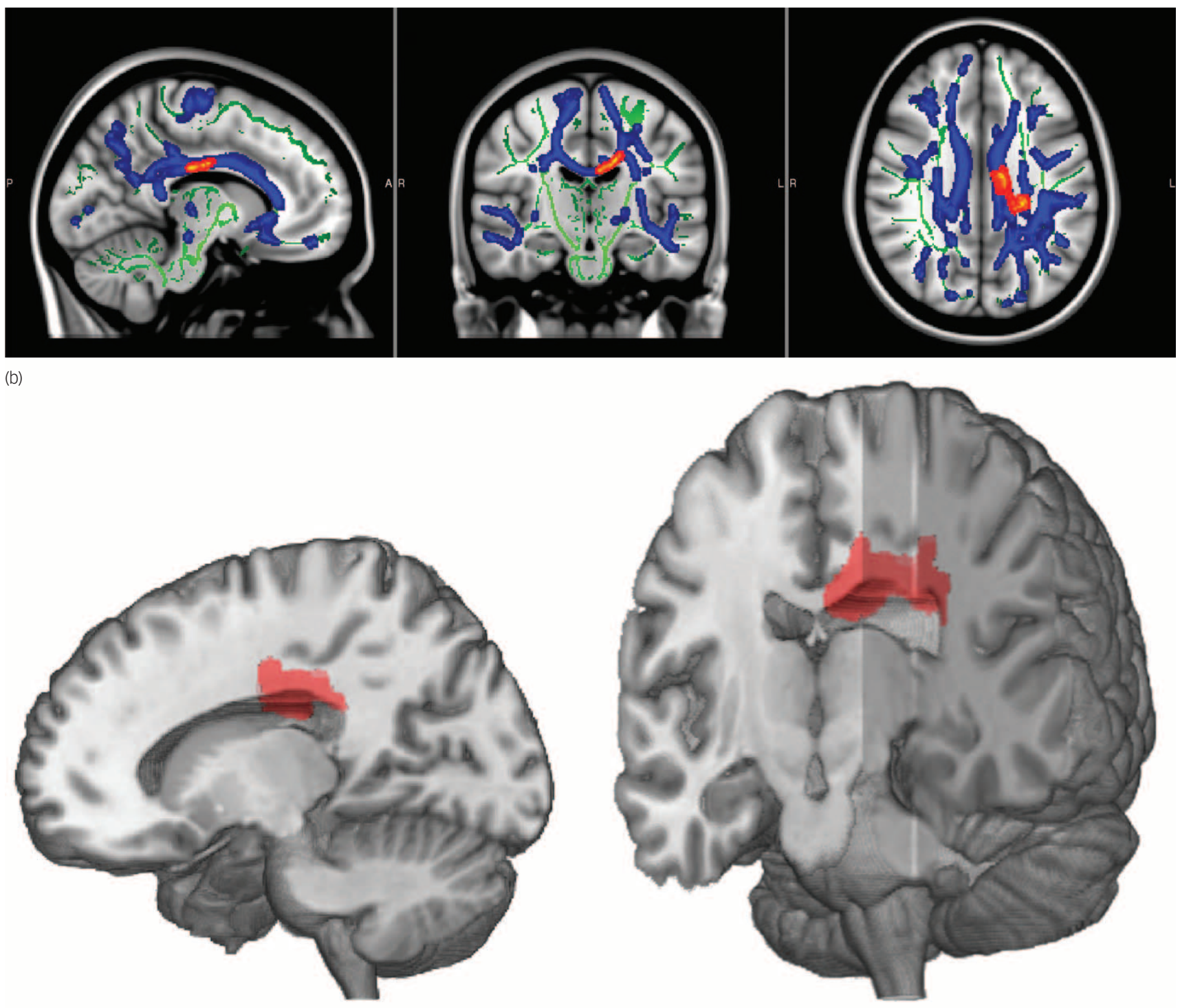

Fig. 1 Results of permutation cluster analysis of fractional anisotropy.

In red ((a) and (b)), regions of significantly reduced fractional anisotropy in the gambling disorder group $v$. control group, after controlling for age. In blue (a), regions of significantly reduced fractional anisotropy in the gambling disorder group $v$. control group, without including age as a covariate.

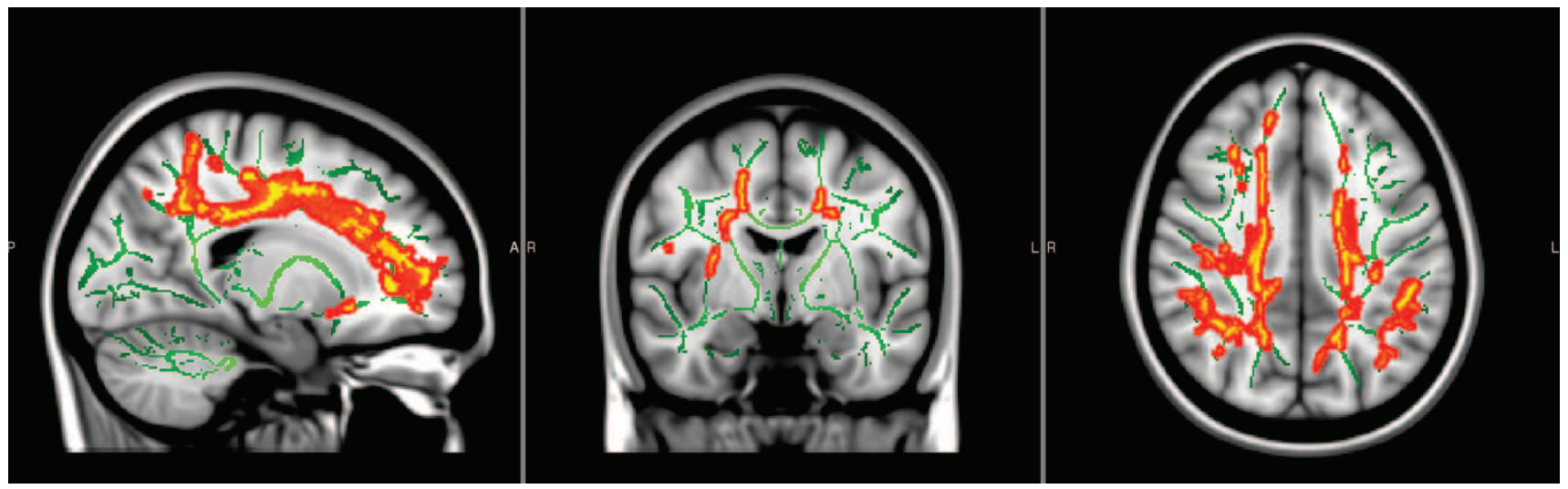

Fig. 2 Results of permutation analysis for clinical measures in the gamblers.

In red, regions in which fractional anisotropy correlated positively with the urge subscale of the Yale-Brown Obsessive Compulsive Scale modified for Pathological Gambling $(P<0.03)$. 


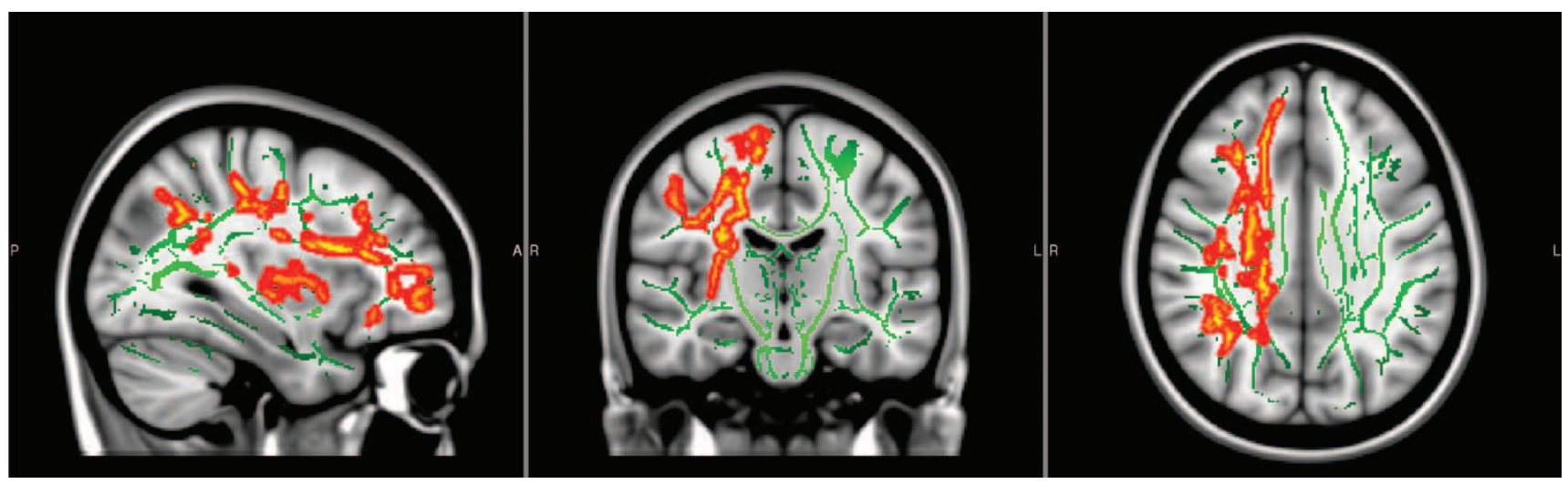

Fig. 3 Results of permutation analysis for clinical measures in the gambling disorder group.

In red, regions in which fractional anisotropy correlated positively with the Gambling Symptom Assessment Scale $(P<0.01)$.

studies, ideally incorporating not just diffusion tensor imaging (DTI) but also functional neuroimaging, including analysis of functional connectivity across salient neural nodes. Future work could include unaffected first-degree relatives of patients to confirm or refute the trait-like nature of the corpus callosum abnormalities.

\section{Limitations}

There are several limitations that should be considered. First, the overall sample size was small and primarily White in ethnic origin. Larger sample sizes with greater diversity would be ideal for future studies in order to provide a more population-representative overview of gambling disorder. Second, the gambling disorder group were significantly older than the healthy control group. Age is known to be an important factor when considering measurements of diffusivity in the brain (see for example Bhagat $\&$ Beaulieu $^{28}$ ). Fractional anisotropy has been found to vary in normal ageing, with the direction of effect and magnitude of such changes varying considerably depending on the region considered. ${ }^{29}$ For example, age has been reported to be negatively correlated with fractional anisotropy in the corpus callosum genu, but not significantly correlated (in either direction) with fractional anisotropy in the corpus callosum body. ${ }^{29}$ Despite the significant age differences, however, the between-group differences in fractional anisotropy in the corpus callosum were evident whether or not age was included as a covariate in the analysis, suggesting that the result was not driven by this potential confound. Future studies, however, should aim to provide a sample of age- and gender-matched controls in order to more robustly address the questions at hand. Another caveat of the current study, relevant to all DTI studies, is that fractional anisotropy can be influenced by a variety of factors apart from myelination, such as crossing fibre tracts. ${ }^{30}$ Although we refer to the reduced fractional anisotropy in gambling disorder as being consistent with reduced structural integrity of white matter tracts, this does necessarily imply an underlying specific structural pathology, such as that seen with advancing age or in the case of demyelinating neurological disorders; put differently, the underlying causes of reduced fractional anisotropy seen here cannot be addressed using the current neuroimaging techniques.

\section{Funding}

This research was supported by a grant from the National Center for Responsible Gaming to J.E.G. and by a grant to S.R.C. from the Academy of Medical Sciences.
Samuel R. Chamberlain, MB/Bchir, PhD, MRCPsych, Department of Psychiatry, University of Cambridge, Cambridge and Cambridge and Peterborough NHS Foundation Trust (CPFT), Cambridge, UK; Katherine Derbyshire, BS, Department of Psychiatry \& Behavioral Neuroscience, University of Chicago, Chicago, Illinois, USA; Richard E. Daws, Msc, Computational, Cognitive \& Clinical Neuroimaging Lab, Imperial College London, London, UK; Brian L. Odlaug, MPH, Department of Public Health, Faculty of Health and Medical Sciences, University of Copenhagen, Copenhagen, Denmark; Eric W. Leppink, BA, Jon E. Grant, JD, MD, MPH, Department of Psychiatry \& Behavioral Neuroscience, University of Chicago, Chicago, Illinois, USA

Correspondence: Jon E. Grant, JD, MD, MPH, Department of Psychiatry \& Behavioral Neuroscience, University of Chicago, Pritzker School of Medicine, 5841 S. Maryland Avenue, MC 3077, Chicago, IL 60637, USA. Email: jongrant@uchicago.edu

First received 12 Nov 2014, final revision 7 Apr 2015, accepted 11 Jun 2015

\section{References}

1 Hodgins DC, Stea JN, Grant JE. Gambling disorders. Lancet 2011; 378 : 1874-84.

2 Black DW, Shaw M, McCormick B, Allen J. Pathological gambling: relationship to obesity, self-reported chronic medical conditions, poor lifestyle choices, and impaired quality of life. Compr Psychiatry 2013; 54: 97-104.

3 Grant JE, Kim SW. Demographic and clinical features of 131 adult pathological gamblers. J Clin Psychiatry 2001; 62: 957-62.

4 Thon N, Preuss UW, Pölzleitner A, Quantschnig B, Scholz H, Kühberger A, et al. Prevalence of suicide attempts in pathological gamblers in a nationwide Austrian treatment sample. Gen Hosp Psychiatry 2014; 36 342-6.

5 Joutsa J, Saunavaara J, Parkkola R, Niemelä S, Kaasinen V. Extensive abnormality of brain white matter integrity in pathological gambling. Psychiatry Res 2011; 194: 340-6.

6 Yip SW, Lacadie C, Xu J, Worhunsky PD, Fulbright RK, Constable RT, et al. Reduced genual corpus callosal white matter integrity in pathological gambling and its relationship to alcohol abuse or dependence. World I Biol Psychiatry 2013; 14: 129-38.

7 Greenberg P, Corey-Lisle PK, Birnbaum H, Marynchenko M, Claxton A. Economic implications of treatment-resistant depression among employees. Pharmacoeconomics 2004; 22: 363-73.

8 American Psychiatric Association. Diagnostic and Statistical Manual of Mental Disorders (5th edn) (DSM-5). APA, 2013.

9 Grant JE, Donahue $\mathrm{CB}$, Odlaug BL, Kim SW, Miller MJ, Petry NM. Imaginal desensitisation plus motivational interviewing for pathological gambling: randomised controlled trial. Br J Psychiatry 2009; 195: 266-7.

10 Grant JE, Kim SW, Hollander E, Potenza MN. Predicting response to opiate antagonists and placebo in the treatment of pathological gambling. Psychopharmacology 2008; 200: 521-7.

11 Pallanti S, DeCaria CM, Grant JE, Urpe M, Hollander E. Reliability and validity of the pathological gambling adaptation of the Yale-Brown ObsessiveCompulsive Scale (PG-YBOCS). J Gambl Stud 2005; 21: 431-43. 
12 Grant JE, Steinberg MA, Kim SW, Rounsaville BJ, Potenza MN. Preliminary validity and reliability testing of a structured clinical interview for pathological gambling. Psychiatry Res 2004; 128: 79-88.

13 Kim SW, Grant JE, Adson DE, Shin YC. Double-blind naltrexone and placebo comparison study in the treatment of pathological gambling. Biol Psychiatry 2001; 49: 914-21.

14 Oei TP, Raylu N, Casey LM. Effectiveness of group and individual formats of a combined motivational interviewing and cognitive behavioral treatment program for problem gambling: a randomized controlled trial. Behav Cogn Psychother 2010; 38: 233-8.

15 Kim SW, Grant JE, Potenza MN, Blanco C, Hollander E. The Gambling Symptom Assessment Scale (G-SAS): a reliability and validity study. Psychiatry Res 2009; 166: 76-84.

16 Sheehan DV, Lecrubier $\mathrm{Y}$, Sheehan $\mathrm{KH}$, Amorim $\mathrm{P}$, Janavs J, Weiller $\mathrm{E}$, et al. The Mini-International Neuropsychiatric Interview (M.I.N.I.): the development and validation of a structured diagnostic psychiatric interview for DSM-IV and ICD-10. J Clin Psychiatry 1998; 59 (suppl 20): 22-33.

17 Hamilton M. A rating scale for depression. J Neurol Neurosurg Psychiatry 1960; 23: 56-62.

18 Sheehan DV. The Anxiety Disease. Scribner, 1983.

19 Frisch MB, Cornell J, Vliianueva M, Retzlaff PJ. Clinical validation of the Quality of Life Inventory. A measure of life satisfaction for use in treatment planning and outcome assessment. Psychol Assess 1992; 4: 92-101.

20 Smith SM, Jenkinson M, Woolrich MW, Beckmann CF, Behrens TE, Johansen-Berg $\mathrm{H}$, et al. Advances in functional and structural MR image analysis and implementation as FSL. Neuroimage 2004; 23 (suppl 1): S208-19.

21 Smith SM, Jenkinson M, Johansen-Berg $H$, Rueckert D, Nichols TE, Mackay CE, et al. Tract-based spatial statistics: voxelwise analysis of multi-subject diffusion data. Neuroimage 2006; 31: 1487-505.
22 Koch K, Reess TJ, Rus OG, Zimmer C, Zaudig M. Diffusion tensor imaging (DTI) studies in patients with obsessive-compulsive disorder (OCD): a review. J Psychiatr Res 2014; 54: 26-35.

23 Grant JE, Correia S, Brennan-Krohn T. White matter integrity in kleptomania: a pilot study. Psychiatry Res 2006; 147: 233-7.

24 Chamberlain SR, Hampshire A, Menzies LA, Garyfallidis E, Grant JE, Odlaug $\mathrm{BL}$, et al. Reduced brain white matter integrity in trichotillomania: a diffusion tensor imaging study. Arch Gen Psychiatry 2010; 67: 965-71.

25 Salo R, Nordahl TE, Buonocore $\mathrm{MH}$, Natsuaki $\mathrm{Y}$, Waters $\mathrm{C}$, Moore $\mathrm{CD}$, et al. Cognitive control and white matter callosal microstructure in methamphetamine-dependent subjects: a diffusion tensor imaging study. Biol Psychiatry 2009; 65: 122-8.

26 King AV, Linke J, Gass A, Hennerici MG, Tost $\mathrm{H}$, Poupon C, et al. Microstructure of a three-way anatomical network predicts individual differences in response inhibition: a tractography study. Neuroimage 2012; 59: 1949-59.

27 Goudriaan $A E$, Yücel $M$, van Holst RJ. Getting a grip on problem gambling: what can neuroscience tell us? Front Behav Neurosci 2014; 8: 141.

28 Bhagat YA, Beaulieu C. Diffusion anisotropy in subcortical whitematter and cortical gray matter: changes with aging and the role of CSF-suppression. J Magn Reson Imaging 2004; 20: 216-27.

29 Yang $Y$, Bender AR, Raz N. Age related differences in reaction time components and diffusion properties of normal-appearing white matter in healthy adults. Neuropsychologia 2015; 66: 246-58.

30 Jones DK, Knösche TR, Turner R. White matter integrity, fiber count, and other fallacies: the do's and don'ts of diffusion MRI. Neuroimage 2013; 73 239-54. 